\title{
A Desirability Function-Based Relatively Optimal Interval Core Model and an Algorithm for Fuzzy Profit Allocation Problems of Enterprise Strategy Alliance
}

\author{
Fei Guan* \\ School of Management and Economics, Beijing Institute of Technology, Beijing, 100081, China \\ E-mail: guanfei@bit.edu.cn \\ Qiang Zhang \\ School of Management and Economics, Beijing Institute of Technology, Beijing, 100081, China \\ E-mail: qiangzhang@bit.edu.cn
}

Received 29 October 2013

Accepted 26 November 2013

\begin{abstract}
Enterprise strategic alliance is a win-win business competition mode that has been developed in both academia and industry. How to allocate total profit fairly in an uncertain environment, however, is an important factor affecting the stability of the strategic alliance. To handle this problem, this paper proposes a new interval number ranking method based on synthesis effect function in which four basic principles to construct an appropriate synthesis effect function are developed. Using the new interval number ranking method, this paper defines a new concept of interval core. The necessary and sufficient condition to guarantee that the interval core is nonempty is also discussed and addressed using a special kind of additive synthesis effect function. A relatively optimal interval core model and an algorithm are developed to solve the proposed fuzzy allocation problem. Finally, a case study is presented to validate the proposed model and algorithm. Data analysis shows that we can obtain the only fuzzy allocation result by choosing an appropriate desirability function. The result indicates that our model has better operability and interpretability than existing methods, and thus can serve as a guideline for developing different fuzzy allocation methods.
\end{abstract}

Keywords: Enterprise strategic alliance; cooperation games; fuzzy cooperation games; fuzzy decision-making; desirability function.

\section{Introduction}

With the globalization of the economy, there is an urgent inclination for most enterprises to broaden their business market. However, many difficulties may occur in this process, such as small scale investment, high management risk, financial difficulties, lack of investment experience and so on. All kinds of risks make it difficult for individual enterprises to survive in the market. Since the concept of strategic alliance was put forward in the late 1970s by Jan Hopland and Roger Nigel, with the aim of improving market competition ability, many enterprises have adopted the strategic alliance mode and achieved great success. Two examples are the new car models developed through the cooperation of the Ford and Mazda motor companies, and the sale of financial software under the alliance of IBM and The Fuji Bank. In fact, strategic alliance [1-2] is a win-win business competition mode for enterprises to achieve a common strategic target, and to maximize the total profit of the alliance. In such an alliance, enterprises will mutually cooperate, take risks and share profits.

However, how to allocate the total profit fairly and reasonably among so many enterprises in a strategic alliance is very important. Suppose there are $M$

* Corresponding author. 
enterprises, a strategic alliance $\mathrm{Q}$ has been already formed after their cooperation with each other, and alliance $\mathrm{Q}$ can achieve the total profit $\mathrm{R}$ ( $\mathrm{R}$ is a positive real number). So how to allocate the total profit $\mathrm{R}$ among $\mathrm{M}$ enterprises is directly related to the lasting and stable development of an alliance Q [3-4]. In order to make all enterprises accept the way of allocation after joining in alliance Q, there is no doubt that two conditions should be satisfied. Firstly, total profit R must be completely allocated. Secondly, for any enterprise in alliance Q, the profit gained from Q must be better than it can own achieve. For example, if enterprise $\mathrm{A}$ can get profit $R_{1}$ by his own ability, and we allocate it $R_{2}$ after it joins Q, if $R_{1} \geq R_{2}$, that is: the profit he get by joining in an alliance is not better than it can own achieve, we can surely say enterprise A will not accept this way of allocation. So in order to get a stable allocation result and to make each enterprise accept this allocation way, we usually need to compare the size relation of any two real numbers $R_{1}$ and $R_{2}$. However, the profit of any alliance usually cannot be expressed by an accurate real number $R$ as a result of external and subjective factors. For example, usually the profit of an alliance is simply expressed as "about 6 million", or "the estimated income is 6 million", etc. A common way is to estimate the range of the profit from both the pessimistic and optimistic point of view, i.e. we use an interval number $\bar{A}=\left[a^{-}, a^{+}\right]$to represent the profit of each alliance. When the profits of each alliance are all interval numbers, we are inevitably to use the size relationship between interval numbers to get a final allocation result. However, the uncertainty of interval number makes it very hard to give an absolutely fair allocation result. At this time, a new interval allocation method is urgently required. However, fuzzy cooperation game [5-9] is a mathematical model that mainly researches on the cooperation and competition phenomenon among players in a fuzzy, uncertain environment using fuzzy set techniques [10-12] and interval valued fuzzy set techniques [13-14]. Its main research focuses on which players can eventually cooperate and how to allocate the profits gained from their cooperation. Interval cooperative game is a special kind of fuzzy cooperative game with the profits of any alliance are interval numbers, interval core and interval Shapley value are very important allocation solution concepts. In interval core and interval Shapley value, all players will accept the allocation result without any opinions, so the solution concepts, models, and theorems in interval cooperative game can serve as a sound basis for research on the above interval profit allocation problems.

In this paper, and given the above background, we mainly discuss the allocation solutions of interval cooperative games when the profits of any alliance are arbitrary interval numbers $\bar{A}_{i}=\left[a_{i}^{-}, a_{i}^{+}\right]$. In recent years, many scholars have conducted extensive researches in order to give a reasonable solution concept to solve this interval profit allocation problem and many successes have been achieved. For example, [15] discussed the convexity of the interval undominated cores for any two interval numbers $\bar{A}=\left[a^{-}, a^{+}\right]$and $\bar{B}=\left[b^{-}, b^{+}\right]$, based on the sequence relation $\bar{A} \leq \bar{B} \Leftrightarrow a^{-} \leq b^{-}, a^{+} \leq b^{+}$, [16] analyzed the interval Shapley value based on the element games constructed by the left and right point $v-$, $v+$ of the interval payoffs, then proved three axioms of Shapley value, [17] defined that the interval Shapley value is the set of Shapley values of element games and proved that the Shapley value of a player $i$ is an interval number, [18] proposed the new superadditivity and researched interval games based on the interval sequence relation $\bar{A} \leq \bar{B} \Leftrightarrow a^{+} \leq b^{-}$, [19] discussed some characterizations of convex interval games by the sequence relation $\bar{A} \leq \bar{B} \Leftrightarrow a^{-} \leq b^{-}, a^{+} \leq b^{+}$, [20] gave the axiom characteristics of convex interval games with sequence relation $\bar{A} \leq \bar{B} \Leftrightarrow a^{-} \leq b^{-}, a^{+} \leq b^{+}$and its new defined subtraction operation, [21] gave the definition of F-core and discussed the necessary condition to guarantee that F-core was nonempty. Based on the above researches, we can easily get that: 1) For any two interval numbers $\bar{A}=\left[a^{-}, a^{+}\right]$and $\bar{B}=\left[b^{-}, b^{+}\right]$, researchers are mainly based on the interval sequence relation $\bar{A} \leq \bar{B} \Leftrightarrow a^{-} \leq b^{-}, a^{+} \leq b^{+}$or $\bar{A} \leq \bar{B} \Leftrightarrow a^{+} \leq b^{-}$. However, these traditional methods are still not very satisfactory $[22,23]$ for decision making process for it is relatively strict by $\bar{A} \leq \bar{B} \Leftrightarrow a^{+} \leq b^{-}$and we cannot compare any two interval numbers such as $\bar{A}=[3,6], \bar{B}=[5,9]$. By $\bar{A} \leq \bar{B} \Leftrightarrow a^{-} \leq b^{-}, a^{+} \leq b^{+}$, it is not a total ordering, sometimes there is no size relation between any two interval numbers such as $\bar{A}=[3,8], \bar{B}=[4,7]$. But we may need to compare the size relation between any two interval numbers while dealing with interval allocation problems. Therefore, it is very important to propose a ranking approach of interval numbers with total ordering and can realize the size relation between any two interval numbers. 2) The exiting solutions in interval 
cooperative games, such as the interval core, interval Shapley value, sometimes do not exist and even if interval core exists, it is not the only solution. It makes it difficult for players with different decision perceptions to make a selection, so how to get the only one interval allocation solution is urgently needed.

The main contributions of this paper are motivated by the above presentation and can be summarized in the following way: 1) We propose a new interval number ranking method based on synthesis effect function, which is the basis of the following researches with a total ordering and can effectively realize the size relation of any two interval number, the construction method of the synthesis effect function is systematically discussed. 2) We propose a new allocation solution concept-interval core using the interval number ranking method and by a special kind of additive synthesis effect function, a theorem to guarantee a nonempty interval core is formulated to solve the allocation problems when profits of each alliance are interval numbers. 3) With the aim to give the only one stable allocation solution, by introducing the definition of desirability function, we construct a relatively optimal interval core model and a relative algorithm to solve the model.

The rest of the paper is structured as follows. Section 2 gives preliminaries, and a new interval number ranking method based on synthesis effect function and some basic principles for constructing synthesis effect function are presented in Section 3. A new concept of interval core, a theorem to guarantee a nonempty interval core by means of a special kind of additive synthesis effect function, and a relatively optimal interval core model, which can give the single allocation result, are shown in Section 4. A case-based example is shown in Section 5. The conclusions and further study are discussed in Section 6.

\section{Preliminaries}

This section will give some preliminaries which are used in the following sections.

\subsection{Interval Numbers and their Operations}

Definition 2.1. ${ }^{[24]}$ Suppose $R$ is the family of all real numbers, for $\forall a^{-}, a^{+} \in R$, if $a^{-} \leq a^{+}$, then we call the bounded closed interval $\bar{A}=\left[a^{-}, a^{+}\right]$an interval number on $R$. In $\bar{A}=\left[a^{-}, a^{+}\right], a^{-}$is called the left endpoint and $a^{+}$is called the right endpoint.
Clearly, for $\forall a \in R$, if we suppose $a$ is the same with $[a, a]$, then we can get that interval numbers are the extension of real numbers. We denote the set of all interval numbers as $I(R)$.

For $\forall \bar{A}=\left[a^{-}, a^{+}\right], \bar{B}=\left[b^{-}, b^{+}\right] \in I(R), k \in R^{+}$, we define the operations of interval numbers as follows:

1) $\bar{A}+\bar{B}=\left[a^{-}+b^{-}, a^{+}+b^{+}\right]$.

2) $\bar{A}=\bar{B} \Leftrightarrow a^{-}=b^{-}, a^{+}=b^{+}$.

3) $k \bar{A}=\left[\mathrm{ka}^{-}, k a^{+}\right]$.

\subsection{Desirability Function}

Desirability function is usually used in optimization process in order to seek a satisfactory solution. It has well applied in many areas, such as engineering design, decision-making, information control and so on. Following, we will give some relative concepts.

Definition 2.2. ${ }^{[25]}$ For a certain optimization problem, a desirability standard $Q$ is used to measure users' satisfaction level with a feasible solution.

Definition 2.3. ${ }^{[25]}$ By a desirability standard Q , only one evaluation result exists for each feasible solution, the set of all evaluation results is denoted by $\mathrm{T}$. If the following relationship exists between the evaluation results of any two feasible solutions, then we call it a desirability sequence.

1) Ordering. For any two feasible solutions $d_{1}$ and $d_{2}, t_{1}$ and $t_{2}$ are the mapping results of $d_{1}$ and $d_{2}$ under $Q$. If $t_{1}>(<,=) t_{2}$, then the desirable degree of $d_{1}$ is higher (lower, equal to) than that of $d_{2}$.

2) Transitivity. For any feasible solutions $d_{1}$, $d_{2}$ and $d_{3}$, if $t_{1} * t_{2}, t_{2} * t_{3}$, then $t_{1} * t_{3} .(*$ is $>,<,=$ etc).

Definition 2.4. ${ }^{[25]}$ For a certain problem optimization $P$, suppose its feasible region is $D, d$ is an arbitrary feasible solution in $D$ and the desirability standard is $Q$. If there exists a mapping $f: D \rightarrow T$, and $T$ has the desirability sequence, then we call $t \in T$ the satisfaction degree of the feasible solution $d$ by the desirability standard $Q$.

\subsection{Concepts of Classical Cooperation Games}

Definition 2.5. ${ }^{[26]}$ A classical cooperation game is usually defined as a pair $\Gamma=(N, v)$, where $N=\{1,2, \cdots, n\}$ is the set of players, $v: 2^{N} \rightarrow R$ is a function assigning to each coalition $S \in 2^{N}$ a real number $v(S) \cdot v(S)$ is called the payoff value of a coalition $S$ with $v(\varnothing)=0$.

We denote by $G^{N}$ the family of all classical cooperative games. In the following, we will use the 
superadditivity of classical cooperation games, which is defined as follows:

Definition 2.6. ${ }^{[26]}$ A game $v \in G^{N}$ is superadditive, if $v$ satisfies:

$$
v(A \bigcup B) \geq v(A)+v(B), \forall A, B \subseteq N, A \cap B=\varnothing
$$

On the research of cooperation games, how to allocate the payoff value $v(S)$ reasonably among each player is the most important issue. Core is one of the most important solution concepts, prior to giving the definition of Core, we will give the definition of imputation.

Definition 2.7. ${ }^{[26]}$ An imputation of a game $v \in G^{N}$ is a n-dimensional vector $x=\left(x_{1}, x_{2}, \cdots, x_{n}\right) \in R^{n}$ satisfying

$$
\begin{gathered}
x_{i} \geq v(\{i\}), i=1,2, \cdots, n \\
\sum_{i=1}^{n} x_{i}=v(N)
\end{gathered}
$$

$x_{i}$ is the profit allocated to player $i$ after their cooperation. $v(\{i\})$ is the profit player $i$ can own achieve. (2) is called the individual rationality, and (3) is called the group rationality.

Definition 2.8. ${ }^{[26]}$ A core of a game $v \in G^{N}$ is a set of ndimensional vector $x=\left(x_{1}, x_{2}, \cdots, x_{n}\right) \in \mathrm{R}^{\mathrm{n}}$ satisfying:

$$
\begin{gathered}
\sum_{i \in R} x_{i} \geq v(R), \forall R \subseteq N \\
\sum_{i=1}^{n} x_{i}=v(N)
\end{gathered}
$$

In the following, in order to simplify the formula, we write $v(i)$ instead of $v(\{i\})$, and $x(R)$ instead of $\sum_{i \in R} x_{i}$. The above symbols and definitions will be used in the following sections.

From Definition 2.8, we can easily see that sometimes there may not exist one solution $x=\left(x_{1}, x_{2}, \cdots, x_{n}\right) \in \mathrm{R}^{\mathrm{n}}$ which satisfies (4) and (5) simultaneously, that is, the core is usually an empty set, see the following example.

Example 2.1 Let $(N, v)$ be a three-person cooperation game. If we know the following: $v(i)=0,(i=1,2,3)$, $v(1,2)=0.8, \quad v(1,3)=0.75, v(2,3)=0.6, v(1,2,3)=1$, then we can get the core by Definition 2.8 . Suppose $x_{1}, x_{2}, x_{3}$ is the core, then $x_{1}, x_{2}, x_{3}$ should satisfy (4) and (5) simultaneously:

$$
\left\{\begin{array}{l}
x_{1} \geq 0 \\
x_{2} \geq 0 \\
x_{3} \geq 0 \\
x_{1}+x_{2} \geq 0.8 \\
x_{2}+x_{3} \geq 0.6 \\
x_{1}+x_{3} \geq 0.75 \\
x_{1}+x_{2}+x_{3}=1
\end{array}\right.
$$

Solving the above system of inequalities, we can easily see that (6) has no solution, so core $x_{1}, x_{2}, x_{3}$ does not exist.

\section{A Synthesis Effect-Based Interval Number Ranking Method}

In order to solve the above allocation problems with the profits of any alliance are interval numbers, we inevitably need to compare the size relations between two interval numbers. Interval numbers are a special kind of set of real numbers and there is no definite size relation for interval numbers. A problem of obtaining a reasonable interval number ranking method is therefore important.

\subsection{Compound Quantification Description Modes of Uncertain Information}

Definition 3.1. ${ }^{[27]}$ Suppose $H$ represents a kind of uncertain information. If the real number $h$ is the principal indicator to describe uncertain information $H$ in some sense, and the real numbers $h_{1}, h_{2}, \cdots, h_{t}$ denote the auxiliary indicator describing the connections between $h$ and $H$ from different angles, then we call the total information $\left(h, h_{1}, h_{2}, \cdots, h_{t}\right)$ constituted by $h$ and $h_{1}, h_{2}, \cdots, h_{t}$ a compound quantification value of $H$.

Take an interval number $\bar{A}=\left[a^{-}, a^{+}\right]$as an example. If we use $M(\bar{A})$ to denote the principal indicator of $\bar{A}=\left[a^{-}, a^{+}\right], U(\bar{A})$ to denote the auxiliary indicators reflecting whether $M(\bar{A})$ can represent interval number $\bar{A}$, then $(M(\bar{A}), U(\bar{A}))$ is a compound quantification mode of $\bar{A}=\left[a^{-}, a^{+}\right]$. In practice, we can choose different compound quantification modes according to different decision environment. For instance, $\left(a^{-} ; a^{+}-a^{-}\right)$is a conservative compound quantification mode of $\bar{A}=\left[a^{-}, a^{+}\right],\left(a^{+} ; a^{+}-a^{-}\right)$is a risky compound quantification mode, and $\left(\left(a^{-}+a^{+}\right) / 2 ; a^{+}-a^{-}\right) \quad$ is a neutral compound quantification mode. In this paper, we will choose $\left(a^{-}, a^{+}-a^{-}\right)$as the compound quantification mode, (namely $M(\bar{A})=a^{-}$represents the principal 
indicator of $\bar{A}=\left[a^{-}, a^{+}\right]$and $U(\bar{A})=a^{+}-a^{-}$represents the auxiliary indicator describing the uncertainty when $M(\bar{A})$ stands for $\bar{A}=\left[a^{-}, a^{+}\right]$. It is easily to see that:

$$
\text { 1) } \begin{array}{ll} 
& M(\bar{A}+\bar{B})=M(\bar{A})+M(\bar{B}) \\
& U(\bar{A} \pm \bar{B})=U(\bar{A})+U(\bar{B}), \bar{A}, \bar{B} \in I(R) \\
\text { 2) } & M(k \bar{A})=k M(\bar{A}) \\
& U(k \bar{A})=k U(\bar{A}), k \in[0,+\infty), \bar{A} \in I(R)
\end{array}
$$

\subsection{Basic Principles for Constructing Synthesis Effect Function}

With the compound quantification mode of interval numbers, if all indexes of an interval number can be synthesized to a synthesis effect value which transforms the interval number to a real number, then it is easy and effective to compare interval numbers. However, choosing a suitable synthesis effect function is very important. Below we will propose some basic principles for choosing the synthesis effect function.

Principle 1. The synthesis effect value is not less than the left endpoint, and is not bigger than the right endpoint.

Principle 2. When the left endpoints of two interval numbers are the same, the synthesis effect value will not decrease as the length of interval increases.

Principle 3. When the lengths of intervals are the same for both numbers, the synthesis effect value will not decrease as the left endpoint increases.

Principle 4. For interval numbers $\left[a^{-}, a^{+}\right]$and $\left[b^{-}, b^{+}\right]$, when $a^{+} \leq b^{-}$, the synthesis effect value of $\left[a^{-}, a^{+}\right]$is not bigger than that of $\left[b^{-}, b^{+}\right]$.

The above principles are the basic conditions for constructing the synthesis effect function. If we adopt $(M(\bar{A}), U(\bar{A}))=\left(a^{-} ; a^{+}-a^{-}\right) \quad$ as the compound quantification mode of $\bar{A}=\left[a^{-}, a^{+}\right]$, we can combine $M(\bar{A})$ and $U(\bar{A})$ by a binary function $L(x, y)$ defined on $\mathrm{R} \times[0, \infty) \rightarrow \mathrm{R}$ (here, $x$ represents $M(\bar{A}), y$ represents $U(\bar{A})$ ).

We can get that the above four principles are equivalent to the following conditions:

1) $x \leq L(x, y) \leq x+y$

2) For any $x \in \mathrm{R}, L(x, y)$ is monotone nondecreasing with $y$.

3) For any $y \in[0,+\infty), L(x, y)$ is monotone nondecreasing with $x$.

4) When $x_{1}+y_{1} \leq x_{2}, L\left(x_{1}, y_{1}\right) \leq L\left(x_{2}, 0\right)$.

Proof. For an interval number $\bar{A}=\left[a^{-}, a^{+}\right]$, if we use binary function $L(x, y)$ as the synthesis effect function, and $x$ represents the left endpoint $a^{-}$of $\bar{A}$, $y$ represents the interval length $a^{+}-a^{-}$of $\bar{A}$, then $x+y$ represents the right endpoint. So (1) it is clear that the representation of principle 1 is equivalent to $x \leq L(x, y) \leq x+y ; \quad$ (2) Clearly principle 2 and principle 3 are equivalent to 2) and 3); (3) In principle 4, when $a^{+} \leq b^{-}$, the synthesis effect value of $\left[a^{-}, a^{+}\right]$is not bigger than that of $\left[b^{-}, b^{+}\right]$, that is to say, when $x_{1}+y_{1} \leq x_{2}$, we have $L\left(x_{1}, y_{1}\right) \leq L\left(x_{2}, y_{2}\right)$, since $y \in[0,+\infty), \quad L(x, y)$ is monotone non-decreasing with $y$, so only if $L\left(x_{1}, y_{1}\right) \leq L\left(x_{2}, 0\right)$, we can get $L\left(x_{1}, y_{1}\right) \leq L\left(x_{2}, y_{2}\right)$.

It is easy to prove that

$$
\begin{gathered}
L(x, y)=x+k y, 0 \leq k \leq 1 \\
L(x, y)=x+\ln (1+k y), 0 \leq k \leq 1
\end{gathered}
$$

are the synthesis effect functions satisfying the above four conditions, we call them the general synthesis effect functions.

Remark 3.1. $k$ in (7) and (8) is an important parameter to reflect the role of auxiliary indicator $y, k$ is bigger (smaller), the attention degree of auxiliary indicator is bigger (smaller), if $k=0$, we can ignore the role of auxiliary indicator. In real decision making environment, we can choose different $k$ by different decision requirements.

Clearly, removing condition 1) will not affect the ranking results in any significant way, so if $L(x, y)$ satisfies conditions 2)-4) only, we call it a general weak synthesis effect function. It is easy to prove that

$$
\begin{aligned}
& L(x, y)=\alpha x+\beta y, \alpha>0,0 \leq \beta \leq \alpha \\
& L(x, y)=\mathrm{e}^{x}(1+\alpha y)^{2}, 0 \leq \alpha \leq 0.5
\end{aligned}
$$

are general weak synthesis effect functions.

Proof. Set (9) for example, it is easy to see that for any $x \in \mathrm{R}, y \in[0,+\infty), x \leq L(x, y) \leq x+y$ is not always true, so $L(x, y)$ does not satisfy 1$)$, it is clearly that $L(x, y)$ is monotone non-decreasing with $y$, and for any $y \in[0,+\infty), L(x, y)$ is monotone non-decreasing with $x$, so $L(x, y)$ satisfies 2) and 3), for any $x_{1}, y_{1}, x_{2}, y_{2} \in R$, we can have the following conclusion: $L\left(x_{2}, 0\right)-L\left(x_{1}, y_{1}\right)=\alpha x_{2}-\alpha x_{1}-\beta y_{1}=\alpha\left(x_{2}-x_{1}\right)-\beta y_{1}$, since $y_{1} \leq x_{2}-x_{1}$ and $\alpha>0,0 \leq \beta \leq \alpha$, we get $L\left(x_{2}, 0\right) \geq L\left(x_{1}, y_{1}\right)$, so also $L(x, y)$ satisfies 4). Therefore, (9) is a general weak synthesis effect function, the proof of (10) is the same. 
Theorem 3.1. A function $L(x, y)=x+g(y)$ on $\mathrm{R} \times[0, \infty)$ is a general synthesis effect function if and only if: 1) $g(y)$ is increasing on $[0, \infty)$;2) $0 \leq g(y) \leq y$. Proof. If $L(x, y)=x+g(y)$ is a general synthesis effect function, then by definition of the general synthesis effect function, we know that $g(y)$ is increasing on $[0, \infty), 0 \leq L(0, y)=g(y) \leq y$.

Conversely, if $g(y)$ is increasing on $[0, \infty)$ and $0 \leq g(y) \leq y$, then we can get that $L(x, y)=x+g(y)$ satisfies the following conditions:

1) $x \leq L(x, y) \leq x+y$;

2) When $x_{1}+y_{1} \leq x_{2}, L\left(x_{1}, y_{1}\right)=x_{1}+g\left(y_{1}\right) \leq x_{1}+y_{1}$ $\leq x_{2} \leq y_{2}+g(0)=L\left(y_{2}, 0\right)$;

3) For any given $x \in \mathrm{R}, L(x, y)$ is non-decreasing with $y$;

4) For given $y \in[0,+\infty), L(x, y)$ is non-decreasing with $x$;

5) $L(x, 0)=x+g(0)$ is increasing with $x$. So $L(x, y)=x+g(y)$ is a general synthesis effect function.

Theorem 3.1 provides a way to construct additive synthesis effect functions. If $x$ is interpreted as the principal index of this synthesis effect model, $g(y)$ is the effect value of the uncertainty $y$, then the geometric explanation of the effect function is shown as in Figure 3.1. Although many functions, such as $L(x, y)=x+k y$, $0 \leq k \leq 1 \quad$ or $\quad L(x, y)=x+\ln (1+y) \quad$, satisfy the conditions, very few have simple analytic expressions.

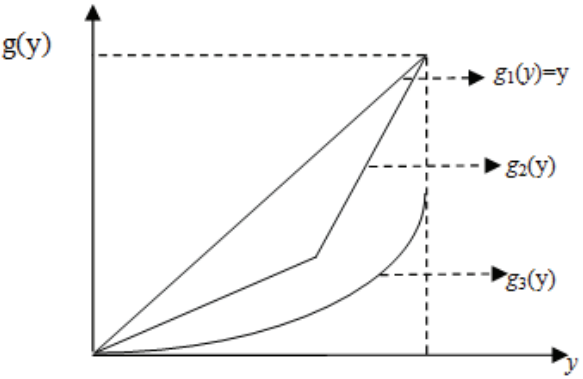

Figure 3.1. The geometric explanation of additive effect metric function

According to the above analyses, for given synthesis effect function $L(x, y)$ and $\bar{A}, \bar{B} \in I(\mathrm{R})$, if we set $L_{(\bar{A})}=L(M(\bar{A}), U(\bar{A}))$ and define the following:

i) $\bar{A}$ is strictly superior to $\bar{B}$, denoted by $\bar{A} \succ \bar{B}$, if and only if

$$
L_{(\bar{A})}>L_{(\bar{B})}
$$

ii) $\bar{A}$ is superior to $\bar{B}$, denoted by $\bar{A} \succ \bar{B}$, if and only if

$$
L_{(\bar{A})} \geq L_{(\bar{B})}
$$

We acquire a ranking method of interval numbers based on synthesis effect function (we will call this SEINRM for short).

The above analysis shows that SE-INRM is the basic mode of interval sequence relation. How to select the appropriate synthesis effect function is very important, but in practice, the selection of synthesis effect function will vary according to different problems and different decision maker's preferences.

\section{A Desirability Function-Based Relatively Optimal Interval Core Model and Algorithm}

\subsection{Interval Core Based on SE-INRM}

Based on SE-INRM and the discussion in Section 3, we can give the order relation between any two interval numbers. We can thus define some basic concepts of interval cooperative games.

Definition 4.1. An interval cooperative game is defined as $(N, \bar{v})$, where $N=\{1,2, \cdots, n\}$ is the set of players, $\bar{v}: 2^{N} \rightarrow I R$ is a function assigning to each coalition $S \in 2^{N}$ a interval number $\bar{v}(S)=\left[v^{-}(S)\right.$, $\left.v^{+}(S)\right] . \quad \bar{v}(S)$ is the fuzzy expected payoff of a coalition, in which $v^{-}(S)$ means the minimum gain of the coalition $S$, and $v^{+}(S)$ means the maximum gain of the coalition $S$ with $\bar{v}(\varnothing)=[0,0]$.

Definition 4.1 clearly expresses that interval cooperative game is a direct extension of classical cooperation games shown in Definition 2.5.

Definition 4.2. In an interval cooperative game $\Gamma=(N, \bar{v})$, based on SE-INRM, if

$$
\bar{v}(A \bigcup B) \succsim \bar{v}(A)+\bar{v}(B), \forall A, B \in N, A \bigcap B=\varnothing
$$

then we call $\Gamma=(N, \bar{v})$ superadditive.

Example 4.1. Let $(N, \bar{v})$ be a three-person interval cooperative game. We know: $\bar{v}(i)=[0,0](i=1,2,3)$, $\bar{v}(1,2)=[8,9], \quad \bar{v}(2,3)=[8,10], \quad \bar{v}(1,3)=[5,15], \quad$ and $\bar{v}(1,2,3)=[10,16]$.

By Definition 4.2, based on SE-INRM, if $L(x, y)=x+k y, k \in[0,1], 1)$ if $k=0$, it is easy to verify that $(N, \bar{v})$ is superadditive; 2) if $k=1$, then $(N, \bar{v})$ is superadditive; 3$)$ if $k=0.5$, then $(N, \bar{v})$ is superadditive.

Definition 4.3. In an interval cooperative game $\Gamma=(N, \bar{v})$, based on SE-INRM, if n-dimensional interval vector $\bar{x}=\left(\bar{x}_{1}, \bar{x}_{2}, \cdots, \bar{x}_{n}\right)$ satisfies: 


$$
\begin{gathered}
\sum_{i=1}^{n} \bar{x}_{i}=\bar{v}(N) \\
\bar{x}_{i} \succsim \bar{v}(i), i=1,2, \cdots, n
\end{gathered}
$$

then we call $\bar{x}=\left(\bar{x}_{1}, \bar{x}_{2}, \cdots, \bar{x}_{n}\right)$ an interval imputation of $\Gamma=(N, \bar{v})$.

Example 4.2. Relative data are the same as example 4.1.

1) if $k=0$, and by the above Definition 4.3, $\bar{x}_{1}=\left[a^{-}, a^{+}\right], \bar{x}_{2}=\left[b^{-}, b^{+}\right], \bar{x}_{3}=\left[c^{-}, c^{+}\right] \quad$ is an interval imputation if the following conditions are satisfied:

$$
\left\{\bar{x}_{1}, \bar{x}_{2}, \bar{x}_{3} \mid a^{-} \geq 0, b^{-} \geq 0, c^{-} \geq 0, a^{-}+b^{-}+c^{-}=10\right\} .
$$

2) If $k=1, \bar{x}_{1}=\left[a^{-}, a^{+}\right], \bar{x}_{2}=\left[b^{-}, b^{+}\right], \bar{x}_{3}=\left[c^{-}, c^{+}\right]$is an interval imputation if the following conditions are satisfied:

$\left\{\bar{x}_{1}, \bar{x}_{2}, \bar{x}_{3} \mid a^{+} \geq 0, b^{+} \geq 0, c^{+} \geq 0, a^{+}+b^{+}+c^{+}=16\right\}$.

3) $k=0.5, \bar{x}_{1}=\left[a^{-}, a^{+}\right], \bar{x}_{2}=\left[b^{-}, b^{+}\right], \bar{x}_{3}=\left[c^{-}, c^{+}\right]$is an interval imputation if the following conditions are satisfied:

$$
\begin{aligned}
& \left\{\bar{x}_{1}, \bar{x}_{2}, \bar{x}_{3} \mid 0.5 a^{-}+0.5 a^{+} \geq 0,0.5 b^{-}+0.5 b^{+} \geq 0,\right. \\
& 0.5 c^{-}+0.5 c^{+} \geq 0, \\
& \left.0.5\left(a^{-}+b^{-}+c^{-}\right)+0.5\left(a^{+}+b^{+}+c^{+}\right)=13\right\} .
\end{aligned}
$$

Definition 4.4. In an interval cooperative game $\Gamma=(N, \bar{v})$, based on SE-INRM, if $\bar{x}=\left(\bar{x}_{1}, \bar{x}_{2}, \cdots, \bar{x}_{n}\right)$ satisfies

$$
\left.C(\bar{v})=\left\{\left(\bar{x}_{1}, \bar{x}_{2}, \cdots, \bar{x}_{n}\right) \in I R^{n} \mid \sum_{i=1}^{n} \bar{x}_{i}=\bar{v}(N), \sum_{i \in S \neq \varnothing} \bar{x}_{i} \succsim \bar{v}(S), \forall S \subseteq N\right)\right\}
$$

then we call $C(\bar{v})$ the interval core of $\Gamma=(N, \bar{v})$.

Example 4.3. Relative data are the same as in Example 4.1 .

1) if $k=0$, by Definition 4.4, $\bar{x}_{1}=\left[a^{-}, a^{+}\right]$, $\bar{x}_{2}=\left[b^{-}, b^{+}\right], \bar{x}_{3}=\left[c^{-}, c^{+}\right]$is an interval core if the following conditions are satisfied:

$\left\{\bar{x}_{1}, \bar{x}_{2}, \bar{x}_{3} \mid a^{-} \geq 0, b^{-} \geq 0, c^{-} \geq 0, a^{-}+b^{-} \geq 8\right.$, $\left.b^{-}+c^{-} \geq 8, a^{-}+c^{-} \geq 5, a^{-}+b^{-}+c^{-}=10\right\}$

2) If $k=1$, by Definition 4.4, $\bar{x}_{1}=\left[a^{-}, a^{+}\right]$, $\bar{x}_{2}=\left[b^{-}, b^{+}\right], \bar{x}_{3}=\left[c^{-}, c^{+}\right]$is an interval core if the following conditions are satisfied:

$$
\begin{aligned}
& \left\{\bar{x}_{1}, \bar{x}_{2}, \bar{x}_{3} \mid a^{+} \geq 0, b^{+} \geq 0, c^{+} \geq 0, a^{+}+b^{+} \geq 9,\right. \\
& \left.b^{+}+c^{+} \geq 10, a^{+}+c^{+} \geq 15, a^{+}+b^{+}+c^{+}=16\right\}
\end{aligned}
$$

3) If $k=0.5$, by Definition 4.4, $\bar{x}_{1}=\left[a^{-}, a^{+}\right], \bar{x}_{2}=\left[b^{-}, b^{+}\right], \bar{x}_{3}=\left[c^{-}, c^{+}\right] \quad$ is an interval core if the following conditions are satisfied:

$$
\left\{\begin{array}{l}
0.5 a^{-}+0.5 a^{+} \geq 0 \\
0.5 b^{-}+0.5 b^{+} \geq 0 \\
0.5 c^{-}+0.5 c^{+} \geq 0 \\
0.5\left(a^{-}+b^{-}\right)+0.5\left(a^{+}+b^{+}\right) \geq 8.5 \\
0.5\left(b^{-}+c^{-}\right)+0.5\left(b^{+}+c^{+}\right) \geq 9 \\
0.5\left(a^{-}+c^{-}\right)+0.5\left(a^{+}+c^{+}\right) \geq 10 \\
0.5\left(a^{-}+b^{-}+c^{-}\right)+0.5\left(a^{+}+b^{+}+c^{+}\right)=13
\end{array}\right.
$$

From Definition 4.4, we can see that interval core is a direct extension of classical core. The same as classical core, interval core always does not exist. So in the following, we will give the sufficient and necessary condition to guarantee a nonempty interval core by a special kind of additive synthesis effect function.

Theorem 4.1. In interval games $\Gamma=(N, \bar{v})$, suppose $A_{i}=\left[a_{i}^{-}, a_{i}^{+}\right]$are the interval core. If we choose the synthesis effect function $L(x, y)=x+k y, 0 \leq k \leq 1$, then $A_{i}=\left[a_{i}^{-}, a_{i}^{+}\right]$is nonempty if and only if: $A_{i}=\left[{a_{i}}^{-}, a_{i}^{+}\right]$satisfies $\sum_{i=1}^{n} a_{i}^{+}=\bar{v}(N)^{+}, \sum_{i=1}^{n} a_{i}^{-}=\bar{v}(N)^{-}$, and

(1) When $\bar{v}(S)^{+}-\bar{v}(S)^{-}-\sum_{i \in S}\left(a_{i}^{+}-a_{i}^{-}\right)>0$

1) when $\sum_{i \in S} a_{i}^{+} \geq \bar{v}(S)^{+}, k \in[0,1]$;

$$
\begin{aligned}
& \text { 2)when } 0 \leq \frac{\sum_{i \in S} a_{i}^{-}-\bar{v}(S)^{-}}{\bar{v}(S)^{+}-\bar{v}(S)^{-}-\sum_{i \in S}\left(a_{i}^{+}-a_{i}^{-}\right)} \leq 1, \\
& k \in\left[0, \frac{\sum_{i \in S} a_{i}^{-}-\bar{v}(S)^{-}}{\bar{v}(S)^{+}-\bar{v}(S)^{-}-\sum_{i \in S}\left(a_{i}^{+}-a_{i}^{-}\right)}\right] ;
\end{aligned}
$$

(2) When $\bar{v}(S)^{+}-\bar{v}(S)^{-}-\sum_{i \in S}\left(a_{i}^{+}-a_{i}^{-}\right)<0$

1) when $0 \leq \frac{\sum_{i \in S} a_{i}^{-}-\bar{v}(S)^{-}}{\bar{v}(S)^{+}-\bar{v}(S)^{-}-\sum_{i \in S}\left(a_{i}^{+}-a_{i}^{-}\right)} \leq 1$, $k \in\left[\frac{\sum_{i \in S} a_{i}^{-}-\bar{v}(S)^{-}}{\bar{v}(S)^{+}-\bar{v}(S)^{-}-\sum_{i \in S}\left(a_{i}^{+}-a_{i}^{-}\right)}, 1\right] ;$ 
2) when $\sum_{i \in S} a_{i}^{+} \geq \bar{v}(S)^{+}, k \in[0,1]$

(3) When $\bar{v}(S)^{+}-\bar{v}(S)^{-}-\sum_{i \in S}\left(a_{i}^{+}-a_{i}^{-}\right)=0, k \in[0,1]$, and $\sum_{i \in S} a_{i}^{-} \geq \bar{v}(S)$

Proof. We clearly know from Definition 4.4 that interval core $A_{i}=\left[a_{i}^{-}, a_{i}^{+}\right]$is nonempty if and only if the inequality constraint $\sum_{i \in S}\left[a_{i}^{-}+k\left(a_{i}^{+}-a_{i}^{-}\right)\right] \geq$ $\bar{v}(S)^{-}+k\left[\bar{v}(S)^{+}-\bar{v}(S)^{-}\right], S \subseteq N$ is satisfied and the equality constraint $\sum_{i=1}^{n}\left[a_{i}^{-}+k\left(a_{i}^{+}-a_{i}^{-}\right)\right]=\bar{v}(N)$ $+k\left(\bar{v}(N)^{+}-\bar{v}(N)^{-}\right)$is satisfied in the feasible solutions. Equality constraint has no relationship with $k$, so we only need to consider the inequality constraints. We simplify the inequality constraints and we obtain

(1) when $\bar{v}(S)^{+}-\bar{v}(S)^{-}-\sum_{i \in S}\left(a_{i}^{+}-a_{i}^{-}\right)>0$

$k \leq \frac{\sum_{i \in S} a_{i}^{-}-\bar{v}(S)^{-}}{\bar{v}(S)^{+}-\bar{v}(S)^{-}-\sum_{i \in S}\left(a_{i}^{+}-a_{i}^{-}\right)}, S \subseteq N, S \neq \varnothing$,

Thus, 1) when $\sum_{i \in S} a_{i}^{+} \geq \bar{v}(S)^{+}$, for $\forall k \in[0,1]$, the above inequality is right, so the interval core is nonempty.

2) when $0 \leq \frac{\sum_{i \in S} a_{i}^{-}-\bar{v}(S)^{-}}{\bar{v}(S)^{+}-\bar{v}(S)^{-}-\sum_{i \in S}\left(a_{i}^{+}-a_{i}^{-}\right)} \leq 1$, for $k \in\left[0, \frac{\sum_{i \in S} a_{i}^{-}-\bar{v}(S)^{-}}{\bar{v}(S)^{+}-\bar{v}(S)^{-}-\sum_{i \in S}\left(a_{i}^{+}-a_{i}^{-}\right)}\right], \quad$ the above inequality is right, so the interval core is nonempty.

3) when $\frac{\sum_{i \in S} a_{i}^{-}-\bar{v}(S)^{-}}{\bar{v}(S)^{+}-\bar{v}(S)^{-}-\sum_{i \in S}\left(a_{i}^{+}-a_{i}^{-}\right)} \leq 0$, for

$\forall k \in[0,1]$, the above inequality is not right, so the interval core is empty.

(2) when $\bar{v}(S)^{+}-\bar{v}(S)^{-}-\sum_{i \in S}\left(a_{i}^{+}-a_{i}^{-}\right)<0$, we get

$$
k \geq \frac{\sum_{i \in S} a_{i}^{-}-\bar{v}(S)^{-}}{\bar{v}(S)^{+}-\bar{v}(S)^{-}-\sum_{i \in S}\left(a_{i}^{+}-a_{i}^{-}\right)}, S \subseteq N, S \neq \varnothing
$$

Thus, 1) when $\sum_{i \in S} a_{i}^{+}<\bar{v}(S)^{+}$, the above inequality is not right, so the interval core is empty.
2) when $0 \leq \frac{\sum_{i \in S} a_{i}^{-}-\bar{v}(S)^{-}}{\bar{v}(S)^{+}-\bar{v}(S)^{-}-\sum_{i \in S}\left(a_{i}^{+}-a_{i}^{-}\right)} \leq 1$, if $k \in\left[\frac{\sum_{i \in S} a_{i}^{-}-\bar{v}(S)^{-}}{\bar{v}(S)^{+}-\bar{v}(S)^{-}-\sum_{i \in S}\left(a_{i}^{+}-a_{i}^{-}\right)}, 1\right], \quad$ the above inequality is right, so the interval core is nonempty.

3) when $\sum a_{i}^{+} \geq \bar{v}(S)^{+}$, for $\forall k \in[0,1]$, the above inequality is not right, so the interval core is empty.

(3) when $\bar{v}(S)^{+}-\bar{v}(S)^{-}-\sum_{i \in S}\left(a_{i}^{+}-a_{i}^{-}\right)=0$, if $\forall k \in[0,1]$ and satisfy $\sum_{i \in S} a_{i}^{-} \geq \bar{v}(S)^{-}$, the above inequality is right, so the interval core is nonempty.

Based on SE-INRM, theorem 4.1 shows the sufficient and necessary condition to guarantee that the interval core is nonempty by a special kind of additive synthesis effect function, which is helpful for us in obtaining the interval core. However, if the interval cores exist, choosing the best one from such a large number is another important issue to consider. In order to solve this problem, following, we will construct a relatively optimal interval core model by the concept of desirability function.

\subsection{A Desirability Function-Based Relatively \\ Optimal Interval Core Model}

From the above analyses we can see if an interval core is nonempty, the selections among so many interval cores will have different tendencies according to different decision environment. To achieve a satisfactory allocation result, we will construct a relative optimum interval core model by the concept of desirability function.

Suppose there are $n$ players. If there are $t$ groups of feasible solutions to guarantee interval core nonempty, which is expressed as follows:

$$
\text { player1 player } 2 \cdots \text { player } \mathrm{n}
$$

Solution $1\left\{\left[a_{11}{ }^{-}, a_{11}{ }^{+}\right] \quad\left[a_{12}{ }^{-}, a_{12}{ }^{+}\right] \quad \cdots \quad\left[a_{1 n}{ }^{-}, a_{1 n}{ }^{+}\right]\right\}$

Solution $\left.2\left\{\left[a_{21}{ }^{-}, a_{21}{ }^{+}\right] \quad\left[a_{22}{ }^{-}, a_{22}{ }^{+}\right] \cdots a_{2 n}{ }^{-}, a_{2 n}{ }^{+}\right]\right\}$

Solution $\operatorname{t~}\left\{\left[a_{t 1}{ }^{-}, a_{t 1}^{+}\right] \quad\left[a_{t 2}{ }^{-}, a_{t 2}{ }^{+}\right] \quad \cdots \quad\left[a_{t n}{ }^{-}, a_{t n}{ }^{+}\right]\right\}$

Based on SE-INRM, suppose the synthesis effect values of each interval solution are the feasible region $\mathrm{D}$, and the evaluation set $T$ is any value belonging to $[0,1]$. We assume $M_{i}$ the biggest synthesis effect value of player $i$, and $m_{i}$ the smallest synthesis effect value of 
player $i$, we then develop the following desirability function.

Definition 4.5. Suppose $\bar{A}_{i}$ is any interval solution of player $i$ in $\mathrm{D}, L_{\left(\bar{A}_{i}\right)}$ is the synthesis effect value of $\bar{A}_{i}$, then we call $P\left(L_{\left(\bar{A}_{i}\right)}, M_{i}, m_{i}\right): D \times R \times R \rightarrow[0,1]$ is a desirability function, if $P\left(L_{\left(\bar{A}_{i}\right)}, M_{i}, m_{i}\right)$ meets the following conditions: (1) $P\left(L_{\left(\bar{A}_{i}\right)}, M_{i}, m_{i}\right)$ is continuous, (2) when $L_{\left(\bar{A}_{i}\right)}=m_{i}, \quad P\left(L_{\left(\bar{A}_{i}\right)}, M_{i}, m_{i}\right)=0$; when $L_{\left(\bar{A}_{i}\right)}=M_{i}, \quad P\left(L_{\left(\bar{A}_{i}\right)}, M_{i}, m_{i}\right)=1$. (3) $P\left(L_{\left(\bar{A}_{i}\right)}, M_{i}, m_{i}\right)$ is monotone non-decreasing with $L_{\left(\bar{A}_{i}\right)}$.

Remark 4.1. Clearly, there may be many different expression forms of desirability function satisfying (1), (2) and (3). Choosing among so many functions will be different by players' different satisfaction levels, desirability function can be in linear form or non-linear form. The geometric explanation can be seen in figure 4.1 .

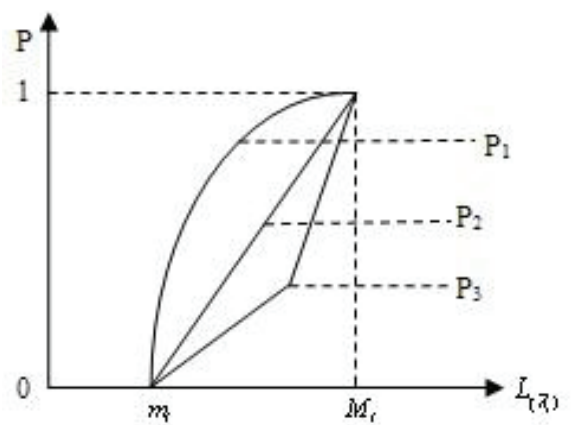

Figure 4.1. The geometric explanation of desirability function

Based on the definition of interval core (see Definition 4.4) and desirability function (see Definition 4.5), we build the following model:

Model 4.1. $\left(\bar{x}_{1}, \bar{x}_{2}, \cdots, \bar{x}_{n}\right)$ is called the relatively optimal interval core based on desirability function, if $\left(\bar{x}_{1}, \bar{x}_{2}, \cdots, \bar{x}_{n}\right)$ is the optimal solution of the following model:

$$
\begin{aligned}
& \max T=\sum_{i=1}^{n} P_{i}\left(L_{\left(\bar{x}_{i}\right)}, M_{i}, m_{i}\right) \\
& \text { s.t. }\left\{\begin{array}{l}
\sum_{i=1}^{n} \bar{x}_{i}=\bar{v}(N), \\
\sum_{i \in S} \bar{x}_{i} \succsim \bar{v}(S), \forall S \subset N
\end{array}\right.
\end{aligned}
$$

Model 4.1 is a relatively optimal interval core model which shows players' satisfaction levels by choosing different desirability functions, if model 4.1 yields a solution $\left(\bar{x}_{1}, \bar{x}_{2}, \cdots, \bar{x}_{n}\right)$, then $\left(\bar{x}_{1}, \bar{x}_{2}, \cdots, \bar{x}_{n}\right)$ will be the most satisfactory interval allocation result.

\subsection{A Relatively Optimal Interval Core Algorithm}

A relatively optimal interval core algorithm is described as follows:

Input: Synthesis effect function $L(x, y)$, desirability functions $P_{i}$, and the payoffs of any coalition $S$.

Output: A relatively optimal interval core, i.e. a relatively optimal interval allocation result.

Step 1: For a given circumstance, select a class of synthesis effect function $L(x, y)$, and set the value of parameter $k$;

Step 2: Set (16) as the constraint conditions, and the biggest (smallest) synthesis effect value of the $i$ th player's imputation interval as the objective function. Compute the biggest (smallest) synthesis effect value of the $i$ th player's imputation interval $M_{i}\left(m_{\mathrm{i}}\right), i=1,2, \cdots, n$;

Step 3: By Definition 4.8, construct the desirability function $P_{i}$;

Step 4: By (17), build the relative optimal interval model;

Step 5: Solve (17) to obtain the solution $\left(\bar{x}_{1}, \bar{x}_{2}, \cdots, \bar{x}_{n}\right)$;

Step 6: Change the value of parameter $k$, and go to step 2-5; we obtain a new solution $\left(\bar{x}_{1}^{\prime}, \bar{x}_{2}^{\prime}, \cdots, \bar{x}_{n}^{\prime}\right)$;

Step 7: According to decision environment in reality and players' different satisfaction levels about the allocation results, choose an appropriate solution $\left(\bar{x}_{1}^{*}, \bar{x}_{2}^{*}, \cdots, \bar{x}_{n}^{*}\right)$, that is the final interval allocation result.

Using the above algorithm, we can easily obtain the allocation result, if we know the payoffs of each coalition, by choosing the appropriate synthesis effect function and desirability function.

\section{A Case-Based Example}

Suppose there are three computer companies A, B, and $\mathrm{C}$. To consolidate market quickly, they decide to cooperative with each other to produce a new production Q. Thanks to their mutual influence and restrictions, they have to cooperate with one another to achieve benefit. If each company produces separately, product Q will not be completed because of technological limitations, for the uncertain factors in reality, the profits may be estimated by interval numbers, that is: if $\mathrm{A}$ and $\mathrm{B}$ cooperate, they can create an income of 1 million to 3 million, if $\mathrm{B}$ and $\mathrm{C}$ cooperate, they can 
create an income 1 million to 2 million, and if $\mathrm{A}$ and $\mathrm{C}$ cooperate, they can create an income of 4 million to 7 million, if all three companies cooperate, they will create an income of 4 million to 8 million. Please give the most satisfactory allocation strategy among the three companies.

According to the analyses, the above problem can be abstracted to the following: if each company produces separately, then $\bar{v}(A)=\bar{v}(B)=\bar{v}(C)=[0,0]$, if $\mathrm{A}$ and $\mathrm{B}$ cooperate, then $\bar{v}(\mathrm{~A}, \mathrm{~B})=[1,3]$, if $\mathrm{B}$ and $\mathrm{C}$ cooperate, then $\bar{v}(B, C)=[1,2]$, if $\mathrm{A}$ and $\mathrm{C}$ cooperate, then $\bar{v}(A, C)=[4,7]$, and if all three companies cooperate, then $\bar{v}(A, B, C)=[4,8]$ (unit: million). Suppose $\bar{x}_{A}=\left[a^{-}, a^{+}\right], \bar{x}_{B}=\left[b^{-}, b^{+}\right], \bar{x}_{C}=\left[c^{-}, c^{+}\right]$are the arbitrary allocation results of $\mathrm{A}, \mathrm{B}$ and $\mathrm{C}$, $0 \leq a^{-} \leq a^{+}, 0 \leq b^{-} \leq b^{+}, 0 \leq c^{-} \leq c^{+}$. In order to make companies $\mathrm{A}, \mathrm{B}$ and $\mathrm{C}$ accept the final allocation result, by Section 4.3, we choose the different synthesis effect functions and desirability functions to have the following tests:

Test $1 L(x, y)=x+k y, 0 \leq k \leq 1$;

$$
P_{i}=\left(L_{\left(\bar{x}_{i}\right)}-m_{i}\right) /\left(M_{i}-m\right) ;
$$

Test $2 L(x, y)=x+k y, 0 \leq k \leq 1$;

$$
P_{i}=\left\{\begin{array}{l}
0, \quad L_{\left(\bar{x}_{i}\right)} \leq \frac{M_{i}+m_{i}}{2} \\
\frac{L_{\left(\bar{x}_{i}\right)}-m_{i}}{M_{i}-m_{i}}, \quad \frac{M_{i}+m_{i}}{2} \leq L_{\left(\bar{x}_{i}\right)} \leq M_{i}
\end{array}\right.
$$

Test $3 L(x, y)=x+\ln (1+k y) ; P_{i}=\left(L_{\left(\bar{x}_{i}\right)}-m_{i}\right) /\left(M_{i}-m\right)$;

Test $4 L(x, y)=x+\ln (1+k y), 0 \leq k \leq 1$;

$$
P_{i}=\left\{\begin{array}{l}
0, \quad L_{\left(\bar{x}_{i}\right)} \leq \frac{M_{i}+m_{i}}{2} \\
\frac{L_{\left(\bar{x}_{i}\right)}-m_{i}}{M_{i}-m_{i}}, \quad \frac{M_{i}+m_{i}}{2} \leq L_{\left(\bar{x}_{i}\right)} \leq M_{i}
\end{array}\right.
$$

We choose a special kind of synthesis effect function as an example. The solving process looks as follows:

Step 1: Select a class of synthesis effect function $L(x, y)=x+k y$, and set the value of parameter $k=0.1$;

Step 2: Set the following (18) as the constraint conditions:

$$
\left\{\begin{array}{l}
{\left[a^{-}, a^{+}\right] \succsim[0,0]} \\
{\left[b^{-}, b^{+}\right] \succsim[0,0]} \\
{\left[c^{-}, c^{+}\right] \succsim[0,0]} \\
{\left[a^{-}, a^{+}\right]+\left[b^{-}, b^{+}\right] \succsim[1,3]} \\
{\left[b^{-}, b^{+}\right]+\left[c^{-}, c^{+}\right] \succsim[1,2]} \\
{\left[a^{-}, a^{+}\right]+\left[c^{-}, c^{+}\right] \succsim[4,7]} \\
{\left[a^{-}, a^{+}\right]+\left[b^{-}, b^{+}\right]+\left[c^{-}, c^{+}\right]=[4,8]}
\end{array}\right.
$$

Set the biggest (smallest) synthesis effect value of the $i$ th player's $(i=A, B, C)$ allocation interval as the objective function. Solve the above linear programming, we can get the following: $M_{A}=3.3, m_{A}=1.1$, $M_{B}=0.1, m_{B}=1.05 \mathrm{E}-13, M_{C}=3.2, m_{C}=1.1$.

Step 3: By Definition 4.5, we choose the desirability function $P_{i}=\left(L_{\left(\bar{x}_{i}\right)}-m_{i}\right) /\left(M_{i}-m\right)$;

Step 4: By (17), build the relative optimal interval model;

Step 5: Solve the model in step 4, we can get: $\bar{x}_{1}=[2.0047,3.5775], \bar{x}_{2}=[0.0152,0.8631], \bar{x}_{3}=[1.9801,3.5593] ;$

Step 6: Change the value of parameter $k$, and go to step 2-5; we obtain new solutions shown in Table 1;

Step 7: Choose an appropriate interval solution according to players' different desirability and attention degree of auxiliary indicator to obtain the final interval allocation result. For example, if we set that the desirability of each company should be bigger than 0.45 , and when we compare the size relation of any two interval numbers, we mainly focuses on the role of the left endpoint, and have little attention to the uncertainty caused by the length of an interval number, then we can choose $k=0.2$ to determine the interval ranking, and we can get the following interval allocation solution $\left(\bar{x}_{A}, \bar{x}_{B}, \bar{x}_{C}\right)=([2.0123,3.5987],[0.0341,0.8636],[1.9536$ $, 3.5377])$ as the final results. That is, we allocate company A about $[2.0123,3.5987]$ million, company B about $[0.0341,0.8636]$ million, and company $\mathrm{C}$ about [1.9536,3.5377] million. But if we set that the desirability of each company must be bigger than 0.6 , that is, each company will be very satisfied with the result, since the target conflicts and other reasons, there sometimes may not exist any allocation solution.

We can get different allocation results in test 24(shown in Tab. 2-4) for different synthesis effect functions and desirability functions. 
Table 1 Relatively optimum interval core based on desirability function

\begin{tabular}{|c|c|c|}
\hline No. & Parameters & Interval allocation results of companies $\mathrm{A}, \mathrm{B}$, and $\mathrm{C}$ and their desirability \\
\hline Case 1 & $k=0.1$ & $\begin{array}{c}\left(\bar{x}_{A}, \bar{x}_{B}, \bar{x}_{C}\right)=([2.0047,3.5775],[0.0152,0.8631],[1.9801,3.5593]) \\
P_{A}=0.4827, P_{B}=0.9999, P_{C}=0.5173\end{array}$ \\
\hline Case 2 & $k=0.2$ & $\begin{array}{c}\left(\bar{x}_{A}, \bar{x}_{B}, \bar{x}_{C}\right)=([2.0123,3.5987],[0.0341,0.8636],[1.9536,3.5377]) \\
P_{A}=0.4707, P_{B}=1.0, P_{C}=0.5293\end{array}$ \\
\hline Case 3 & $k=0.5$ & $\begin{array}{c}\left(\bar{x}_{A}, \bar{x}_{B}, \bar{x}_{C}\right)=([1.824,3.8499],[0.4999,0.5001],[1.676,3.65]) \\
P_{A}=0.4456, P_{B}=1.0, P_{C}=0.5544\end{array}$ \\
\hline Case 4 & $k=0.8$ & $\begin{array}{c}\left(\bar{x}_{A}, \bar{x}_{B}, \bar{x}_{C}\right)=([1.788,3.7067],[0.5684,0.8579],[1.6436,3.4354]) \\
P_{A}=0.423, P_{B}=1.0, P_{C}=0.577\end{array}$ \\
\hline Case 5 & $k=0.9$ & $\begin{array}{c}\left(\bar{x}_{A}, \bar{x}_{B}, \bar{x}_{C}\right)=([1.8494,3.6749],[0.4638,0.9485],[1.6869,3.3766]) \\
P_{A}=0.419, P_{B}=1.0, P_{C}=0.581\end{array}$ \\
\hline
\end{tabular}

Table 2 Relatively optimal interval core based on desirability function

\begin{tabular}{|c|c|c|}
\hline No. & Parameters & Interval allocation results of companies $\mathrm{A}, \mathrm{B}$, and $\mathrm{C}$ and their desirability \\
\hline Case 1 & $k=0.1$ & $\begin{array}{c}\left(\bar{x}_{A}, \bar{x}_{B}, \bar{x}_{C}\right)=([2.0444,3.6001],[0.0123,0.889],[1.9432,3.5109]) \\
P_{A}=0.5, P_{B}=0.9997, P_{C}=0.5\end{array}$ \\
\hline Case 2 & $k=0.2$ & $\begin{array}{c}\left(\bar{x}_{A}, \bar{x}_{B}, \bar{x}_{C}\right)=([2.0885,3.6459],[0.0303,0.8787],[1.8811,3.4754]) \\
P_{A}=0.5, P_{B}=0.9999, P_{C}=0.5\end{array}$ \\
\hline Case 3 & $k=0.5$ & $\begin{aligned}\left(\bar{x}_{A}, \bar{x}_{B}, \bar{x}_{C}\right)= & ([2.2286,3.7714],[0.0103,0.9897],[1.7611,3.2389]) \\
& P_{A}=0.5, P_{B}=1, P_{C}=0.5\end{aligned}$ \\
\hline Case 4 & $k=0.8$ & $\begin{array}{c}\left(\bar{x}_{A}, \bar{x}_{B}, \bar{x}_{C}\right)=([2.1279,3.968],[0.3063,0.9234],[1.5658,3.1086]) \\
P_{A}=0.5, P_{B}=1, P_{C}=0.5\end{array}$ \\
\hline Case 5 & $k=0.9$ & $\begin{array}{c}\left(\bar{x}_{A}, \bar{x}_{B}, \bar{x}_{C}\right)=([2.0671,3.9925],[0.4035,0.9552],[1.5294,3.0523]) \\
P_{A}=0.5, P_{B}=1, P_{C}=0.5\end{array}$ \\
\hline
\end{tabular}

Table 3 Relatively optimal interval core based on desirability function

\begin{tabular}{|c|c|c|}
\hline No. & Parameters & Interval allocation results of companies $\mathrm{A}, \mathrm{B}$, and $\mathrm{C}$ and their desirability \\
\hline Case 1 & $k=0.1$ & $\begin{array}{r}\left(\bar{x}_{A}, \bar{x}_{B}, \bar{x}_{C}\right)=([2.0512,3.3845],[0.007,1.1216],[1.9418,3.4939]) \\
P_{A}=0.500751155, P_{B}=0.999773394, P_{C}=0.499268005\end{array}$ \\
\hline Case 2 & $k=0.2$ & $\begin{array}{c}\left(\bar{x}_{A}, \bar{x}_{B}, \bar{x}_{C}\right)=([1.9345,3.4763],[0.3359,0.8427],[1.7297,3.681]) \\
P_{A}=5.10 \mathrm{E}-01, P_{B}=1.00 \mathrm{E}+00, P_{C}=4.90 \mathrm{E}-01\end{array}$ \\
\hline Case 3 & $k=0.5$ & $\begin{aligned}\left(\bar{x}_{A}, \bar{x}_{B}, \bar{x}_{C}\right)=([0,1.746],[1.0758,1.2593],[2.9242,4.9947]) \\
P_{A}=0.11295217, P_{B}=0.999984456, P_{C}=0.851121977\end{aligned}$ \\
\hline Case 4 & $k=0.8$ & $\begin{array}{c}\left(\bar{x}_{A}, \bar{x}_{B}, \bar{x}_{C}\right)=([0,1.8062],[1.6122,1.7234],[2.3878,4.4703]) \\
P_{A}=1.84 \mathrm{E}-01, P_{B}=1.00 \mathrm{E}+00, P_{C}=7.05 \mathrm{E}-01\end{array}$ \\
\hline Case 5 & $k=0.9$ & $\begin{aligned}\left(\bar{x}_{A}, \bar{x}_{B}, \bar{x}_{C}\right)= & ([0,1.8176],[1.7631,1.8614],[2.2369,4.321]) \\
& P_{A}=0.179066908, P_{B}=0.995075987, P_{C}=0.652994973\end{aligned}$ \\
\hline
\end{tabular}


Table 4 Relatively optimal interval core based on desirability function

\begin{tabular}{|c|c|c|}
\hline No. & Parameters & Interval allocation results of companies $\mathrm{A}, \mathrm{B}$, and $\mathrm{C}$ and their desirability \\
\hline Case 1 & $k=0.1$ & $\begin{aligned}\left(\bar{x}_{A}, \bar{x}_{B}, \bar{x}_{C}\right)=([2.0495,3.3829],[0.007,1.1216],[1.9434,3.4956]) \\
P_{A}=0.522552066, P_{B}=0.998920858, P_{C}=0.477447499\end{aligned}$ \\
\hline Case 2 & $k=0.2$ & $\begin{array}{c}\left(\bar{x}_{A}, \bar{x}_{B}, \bar{x}_{C}\right)=([1.906,3.4479],[0.3358,0.8426],[1.7582,3.7095]) \\
P_{A}=2.44 \mathrm{E}-01, P_{B}=7.13 \mathrm{E}-01, P_{C}=7.62 \mathrm{E}-01\end{array}$ \\
\hline Case 3 & $k=0.5$ & $\begin{array}{r}\left(\bar{x}_{A}, \bar{x}_{B}, \bar{x}_{C}\right)=([1.6124,3.3713],[0.8965,1.0788],[1.4911,3.5499]) \\
P_{A}=0.50000699, P_{B}=0.845419281, P_{C}=0.500009825\end{array}$ \\
\hline Case 4 & $k=0.8$ & $\begin{aligned}\left(\bar{x}_{A}, \bar{x}_{B}, \bar{x}_{C}\right) & =([1.5346,3.352],[1.054,1.1646],[1.4114,3.4834]) \\
P_{A} & =5.00 \mathrm{E}-01, P_{B}=6.71 \mathrm{E}-01, P_{C}=5.00 \mathrm{E}-01\end{aligned}$ \\
\hline Case 5 & $k=0.9$ & $\begin{aligned}\left(\bar{x}_{A}, \bar{x}_{B}, \bar{x}_{C}\right) & =([1.5338,3.3622],[1.0553,1.153],[1.4109,3.4848]) \\
P_{A} & =0.485701891, P_{B}=0.611802735, P_{C}=0.484759586\end{aligned}$ \\
\hline
\end{tabular}

To verify the effectiveness of our model, the allocation results achieved by other methods are shown in Table 5.

Table 5 Allocation results when compared with other methods

\begin{tabular}{cc}
\hline References & Final interval allocation results of A, B, and C using other methods \\
\hline L. Mallozzi.etc [21] & $\left(\bar{x}_{A}, \bar{x}_{B}, \bar{x}_{C}\right)=([2,3],[0,1][2+\alpha, 4-\alpha]), \alpha \in[0,1]$ \\
Yu and Zhang [18] & $\left(\bar{x}_{A}, \bar{x}_{B}, \bar{x}_{C}\right)=([1.5,4],[-5 / 6,5 / 3],[1 / 2,8 / 3])$ \\
Huang and Wu [17] & $\left(\bar{x}_{A}, \bar{x}_{B}, \bar{x}_{C}\right)=\left\{a^{-}, a^{+}, b^{-}, b^{+}, c^{-}, c^{+} \mid a^{-}+b^{-} \geq 1, b^{-}+c^{-} \geq 1, a^{-}+c^{-} \geq 4\right.$ \\
& $\left.4 \leq a^{-}+b^{-}+c^{-} \leq 8, a^{-}=a^{+}, b^{-}=b^{+}, c^{-}=c^{+}\right\}$
\end{tabular}

By theoretical analysis and test results shown in Tables 1 to 4 , we can easily draw the following conclusions: 1) Allocation results are closely related to the selection of desirability function and synthesis effect function, and for a certain desirability function, the change of parameter $k$ can lead to different allocation results, which can clearly show players' different decision awareness and different attention degree to the role of auxiliary indicator; 2) In specific circumstances, an interval core and relatively optimal interval core may not exist. Decision makers should then select an appropriate desirability function and synthesis effect function according to the specific situations and the decision maker's preferences; 3) By comparing the results shown in table 5 , we can easily get that: if companies in an alliance have the same preferences to select a parameter $k$ to determine the ranking method, for certain desirability function and synthesis effect function, we can get the only one interval allocation result (see Tables 1-4), and each allocation result can clearly show players' different decision preference, but due to papers [21], [17] and [15], the change in values of parameters $\alpha, a^{-}, b^{-}, c^{-}, a^{+}, b^{+}, c^{+}$, results in numerous allocation results, it is therefore difficult for players to make a right decision on how to allocate the total profit. In paper [18], company $\mathrm{B}$ will get $[-5 / 6,5 / 3]$ million, any real number in this interval is an allocation result, but in fact, we cannot allocate company B a negative number. So based on the above analyses, our method is better than the other methods.

\section{Conclusion and Further Study}

We proposed a new interval number ranking method (SE-INRM) and discussed the basic principles for selecting an appropriate synthesis effect function. Second, using SE-INRM, we defined the interval core 
and proved the necessary and sufficient condition to guarantee that an interval core was nonempty with a specific synthesis effect function. At third, we established a relatively optimal interval core model by introducing the concept of a desirability function. Lastly, we verified the performance and characteristics of our model using a concrete example. The results show that our model enriches the existing theories, and it can be effectively applied in solving profit allocation problems under uncertain environment when various departments or enterprises collaborate together. However, there are also limitations in our model. For example, we do not set the threshold limit $P_{i} \leq \delta_{i}\left(\delta_{i}\right.$ is the threshold value) in model (17). We therefore obtain only the global optimization solution, which may lead to very low desirability for some players. Our future work will focus on how the allocation results change with the changes in a threshold value $\delta$, and how to extend SE-INRM to a fuzzy number ranking method.

\section{Acknowledgments}

This work was supported by the National Natural Science Foundation of China and Specialized Research Fund for the Doctoral Program of Higher Education (71071018, 70801064, 20111101110036).

\section{References}

1. Z. Z. Shi. Enterprise Strategic Alliance. Shanghai Finance and Economics University Press, 2001.

2. T. K. Das, B-S. Teng. A resource-based theory of strategic alliances, Journal of Management, 26(1) (2000).

3. T. K. Das, B-S. Teng. Instabilities of strategic alliances: An internal tensions perspective. Organization Science, 11(1) (2000) 77-101.

4. X. H. Wang, J. Y. Hu. Probe into the stability of the strategic alliance. Technical Economic and Management Research, (4) (2001) 44-45.

5. F. Y. Meng, Q. Zhang, H. Cheng. The Owen value for fuzzy games with a coalition structure. International Journal of Fuzzy Systems, 14(1) (2012) 22-34.

6. M. Tsurumi, T. Tanino, M. Inuiguchi. A Shapley function on a class of cooperative fuzzy games. European Journal of Operational Research, 129(3) (2001) 596-618.

7. M. Mareš. Fuzzy coalition structures. Fuzzy Sets and Systems, 114(1) (2000) 23-33.

8. D. Butnariu. Fuzzy games: A description of the concept. Fuzzy Sets and Systems, 1(3) (1978) 181-192.

9. M. Sakawa, I. Nishizaki. A lexicographical concept in an n-person cooperative fuzzy game. Fuzzy Sets and Systems, 61(3) (1994) 265-275.

10. J. Lu, G. Q. Zhang, D. Ruan, and F. Wu. Multi-objective group decision making: methods, software and applications with fuzzy set techniques, Imperial College Press, London, 2007.
11. H. Y. Zhang, W. X. Zhang, C. L. Mei. Entropy of interval-valued fuzzy sets based on distance and its relationship with similarity measure, Knowledge-Based Systems, 22(6) (2009) 449-454.

12. G. Q. Zhang, J. Lu. Using general fuzzy number to handle uncertainty and imprecision in group decisionmaking. Intelligent Sensory Evaluation, 2004.

13. J. M. Zhan, Dudek W. A., Y. B. Jun. Interval valued $(\in, \in \vee q)$-fuzzy filters of pseudo BL-algebras. Soft Computing, 13(1) (2009) 13-21.

14. Akram M., Dudek W. A. Interval valued fuzzy graphs. Computers \& Mathematics with Application, 61(2) (2011) 289-299.

15. R. Brânzei S, Z. Alparslan-Gök, O. Brânzei. Cooperative games under interval uncertainty: on the convexity of the interval undominated cores, Central European Journal of Operational Research, 19(4) (2011) 523-532.

16. W. Chen, Q. Zhang. Research on Shapley value of fuzzy cooperative games. Journal of Management Sciences, 9(5) (2006) 50-55.

17. L. J. Huang, Q. Z. Wu, Q. Zhang. Solutions of n-person cooperative with interval worth. Chinese Journal of Management Science, 14(Special Issue) (2006) 140-143.

18. X. H. Yu, Q. Zhang. Interval Shapley value for fuzzy cooperative games. Proceedings of the Second International Conference on Game Theory and Applications, (2007) 256261.

19. R. Brânzei, S. Tijs, S. Z. Alparslan-Gök, Some characterizations of convex interval games, AUCO Czech Economic Review, 2 (2008) 219-226.

20. F. Y. Meng, Q. Zhang. The Shapley function for fuzzy cooperative games with interval payoffs. Transactions of Beijing Institute of Technology, 9(31) (2011) 1131-1134.

21. L. Mallozzi, V. Scalzo, S. Tijs. Fuzzy interval cooperative games. Fuzzy Sets and Systems, 165(1) (2011) 98-105.

22. Z. S. Xu, Q. L. Da. Research on method for ranking interval numbers. System Engineering, 19(6) (2001) 9496.

23. P. Sevastianov. Numerical methods for interval and fuzzy number comparison based on the probabilistic approach and Dempster-Shafer theory. Information Science, 177 (21) (2007) 4645-4661.

24. R. Brânzei, S. Z. Alparslan-Gök. Bankruptcy problems with interval uncertainty. Economics Bulletin, 3(56) (2008) 1-10.

25. H. Z. Huang, X. S. Yao, Z. R. Zhou. Multi-objective optimization design of the working unit of loader based on the satisfactory degree theory. Journal of Mechanical Engineering, 39(5) (2003) 97-103.

26. Z. Xie. Game Theory. National University of Defense Technology Press. 2003.

27. F. C. Li, F. Guan, C. X. Jin. A quantity-based fuzzy number ranking method for decision making with uncertainty. International Journal of Uncertainty, Fuzziness and Knowledge-Based Systems, 20(Suppl.1) (2012) 133-146. 